\title{
Experimental investigation of micro injection molding and relevant numerical simulations in 3D case
}

\author{
Zhiqiang Cheng ${ }^{1, a}$, Thierry Barriere ${ }^{2}$, Baosheng Liu ${ }^{1}$, Jean-Claude Gelin² and Gerard Michel $^{2}$ \\ 1 Department of Applied Mechanics, Southwest Jiaotong University, 610031 Chengdu, P.R. China \\ 2 FEMTO-ST Institute, ENSMM, 24 chemin de l'épitaphe, 25030 Besancon, France
}

Received 16 July 2007; accepted 27 September 2007

\begin{abstract}
Micro injection molding is one of the key technologies to produce small and complicated components in various materials, including plastic, metal, ceramics etc. In order to improve product quality and to reduce manufacturing cost, the specified experiments have been realized to analyze the process parameters. A specially designed mould with five channels has been manufactured at ENSMM. The polypropylene is chosen as injected material in micro mould for the sake of its good fluidity and replication ability. The micro injection experiments are accomplished with the use of a micro injection system. For the application of numerical simulation, the viscous behaviors of polypropylene have been measured. A fully vectorial explicit algorithm has been developed and implemented in the FEM software. The simulations of micro injection process are performed with different wall boundary conditions. Comparisons between the simulation results and the experimental ones show that the simulation with slippery wall boundary condition fits well the experimental results.
\end{abstract}

\section{Introduction}

The domain of micro injection represents the important growth in the last five years. Some micro-components with injection volume less than $1 \mathrm{~cm}^{3}$ have been realized with Battenfeld Microsystem ${ }^{\complement}$. For the micro injection molding researches, some works have been accomplished by different authors, including micro injection system [1], micro injection process parameters $[2,3]$ and its feedstock $[4,5]$. In FEMTO-ST Institute, a lot of research works have been realized in PIM field [6-8].

The present paper presents a specially designed mould with five channels manufactured at ENSMM. The polypropylene is chosen as the material injected in micro mould, for the sake of its good fluidity and replication ability [9]. The viscous behaviors of polypropylene have been tested for the application of numerical simulation. The experiments of micro injection are performed on a two-colours Battenfeld ${ }^{\complement} 50$ micro-system. A fully vectorial explicit algorithm has been developed and implemented in the software [10]. This software can be used to analyze the effects of various injection process parameters. By comparisons between the simulation results and the experimental ones, it can be observed that the simulation with slippery wall boundary condition fits well the experiment results.

\footnotetext{
${ }^{a}$ Corresponding author: zqcheng@netease.com
}

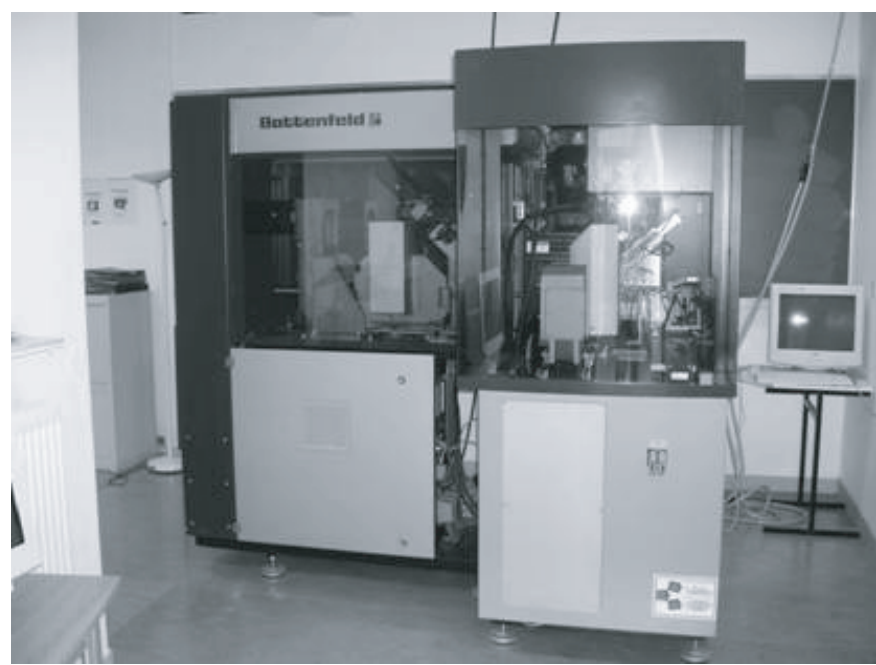

Fig. 1. Two-colours Battenfeld microsystem 50.

\section{Micro injection experiments}

The equipment of two-colours Battenfeld microsystem 50 used in the study is shown in Figure 1. This fully electric production equipment is specially designed for the parts weights as light as $0.1 \mathrm{mg}$. The injection unit is built-up especially for the highly precise injection molding, which includes an extruder screw with $14 \mathrm{~mm}$ diameter, an injection piston with $5 \mathrm{~mm}$ diameter for pre-dosing 


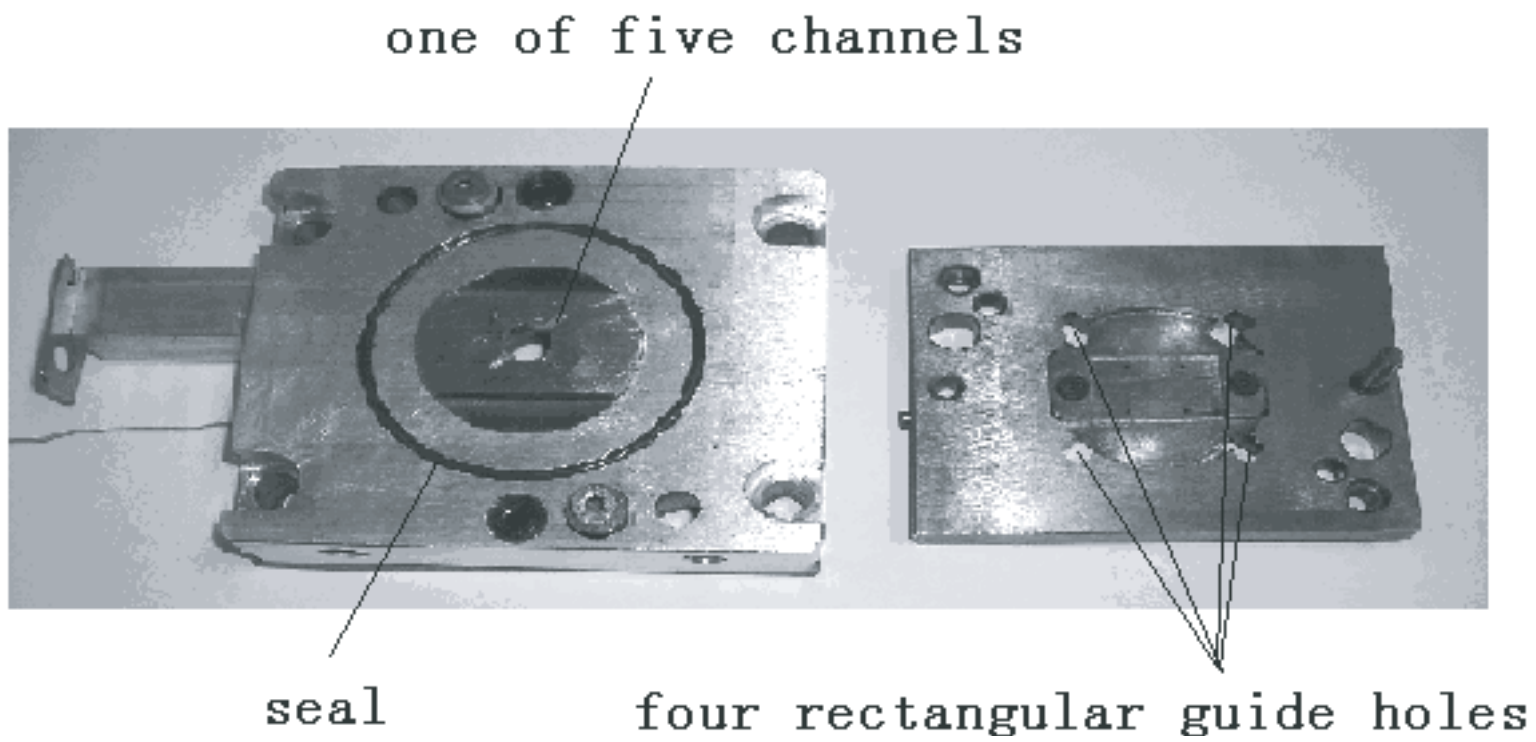

Fig. 2. Mould for the injection experiments.

with a precision of $0.001 \mathrm{~cm}^{3}$. The maximum injection speed is $760 \mathrm{~mm} \cdot \mathrm{s}^{-1}$, the maximum injection pressure is $250 \mathrm{MPa}$ and the theoretical injection volume is from $0.025 \mathrm{~cm}^{3}$ to $1.1 \mathrm{~cm}^{3}$. The maximum clamping force is $50 \mathrm{kN}$ and the ejection force is $1.2 \mathrm{kN}$.

A special mould with 5 channels is manufactured, as shown in Figure 2. The overall dimension of mould cavity is $20 \mathrm{~mm}$, the diameter of central circular part is $10 \mathrm{~mm}$, thickness $0.8 \mathrm{~mm}$. The length of each channel is $5 \mathrm{~mm}$, the width is $1.5 \mathrm{~mm}$. The dimensions of die cavities are measured by a Werth Benchtop ScopeCheck ${ }^{\circledR} 200$ CNC with the use of a Noncontact-Werth Image Processing video sensor of fixed telecentric objective. The four rectangular guides are used for precise assemblage between the fixed die and the movable one. The injection piston can be used as ejector after the injection process.

\section{Polypropylene viscous behaviors}

The polypropylene (PP, EP548N) for injection purpose with $3 \mathrm{~mm}$ average granularity, produced by Basel Company, is used in the experiments. The density of injected polypropylene is $0.73026 \mathrm{~g} \cdot \mathrm{cm}^{3}$, specific heat $2960 \mathrm{~J} \cdot \mathrm{kg}^{-1} \cdot \mathrm{K}^{-1}$ and thermal conductivity $0.16 \mathrm{~W} \cdot \mathrm{m}^{-1} \cdot \mathrm{K}^{-1}$.

The viscosity of polypropylene is tested by a capillary rheometer (Bohlin Instrument), under the shear rates ranging from $1 \mathrm{~s}^{-1}$ to $10^{5} \mathrm{~s}^{-1}$ at different temperatures. The rheometer has a capillary in tungsten carbide material with $1 \mathrm{~mm}$ diameter and $16 \mathrm{~mm}$ length. The tested viscosity is shown in Figure 3. It is to be observed that the polypropylene viscosity is strongly dependent on its temperature, especially when the shear rate is in the range of small values. For example, the viscosity value of polypropylene at $220^{\circ} \mathrm{C}$ is nearly the double of that at $260^{\circ} \mathrm{C}$, when its shear rate is $1 \mathrm{~s}^{-1}$.

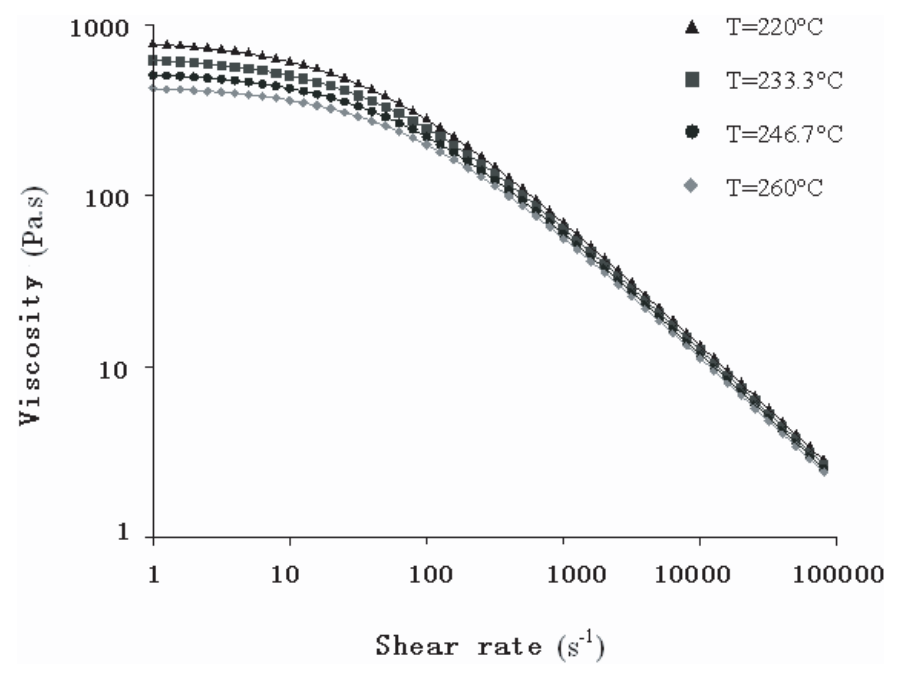

Fig. 3. Polypropylene viscosity versus its shear viscosity obtained by capillary rheometer at different temperatures.

\section{Mechanical modeling of mould filling}

Because of the nature of filling process in micro injection, Eulerian description is used in modeling of the mould filling. Let $t$ be the current instant in injection process: $t \in\left[0, t_{f n}\right], t_{f n}$ represents the final time corresponding to the end of filling process. The whole space in the mould defined as a set $\Omega$, in which the position is represented by the vector $\mathbf{X}$. This set consists of two different parts at each instant: the part filled by feedstock and the remained void part, practically filled by air. They are represented by two adjoined subsets $\Omega^{F}(t)$ and $\Omega^{V}(t)$. A field variable $F(\mathbf{X}, t)$ is used to describe the filling state, which takes value 1 in the filled part and value 0 in the void part.

The injection flow in the filled part of the mould cavity is supposed to be incompressible. It should obey 
the momentum and mass conservation equations. When Reynolds number is small, as in the case of viscous mould filling, the momentum conservation is represented by a Stokes equation. This equation is expressed respectively in the filled and void portion as:

$$
\begin{array}{ll}
\forall \mathbf{X} \in \Omega^{F}, & \rho_{f} \frac{\partial \mathbf{V}}{\partial t}=-\nabla P+\nabla \bullet \mu_{f} \dot{\epsilon}_{f}+\rho_{f} \mathbf{g} \\
\forall \mathbf{X} \in \Omega^{V}, & \rho_{a} \frac{\partial \mathbf{V}}{\partial t}=-\nabla P+\nabla \bullet \mu_{a} \dot{\epsilon}_{a}+\rho_{a} \mathbf{g}
\end{array}
$$

where $\mathbf{V}$ is the velocity vector, $P$ stands for the pressure field, $\mu_{f}$ and $\mu_{a}$ are the viscosities in two different mould parts, $\rho_{f}$ and $\rho_{a}$ their densities in different parts, $\dot{\epsilon}_{f}$ and $\dot{\epsilon}_{a}$ are the strain rate in filled and void part, $\mathrm{g}$ the gravity acceleration vector. To keep the numerical stability, fictive density $\rho_{a}$ and viscosity $\mu_{a}$ corresponding to air are applied respectively in the void mould part.

It should be mentioned that the incompressibility condition is attentively verified only in the filled part, although the same operation is performed in the void part to keep simplicity of the algorithm. By mass conservation, one has the following equation expressed as:

$$
\forall \mathbf{X} \in \Omega^{F}, \quad \nabla \bullet \mathbf{V}=0 .
$$

At each instant $t$ during the injection course, the evolution of filling state variable is governed by an advection equation expressed as:

$$
\forall \mathbf{X} \in \Omega^{F}, \quad \frac{\partial F}{\partial t}+\mathbf{V} \bullet \nabla F=0 .
$$

The feedstock viscous behaviours are strongly dependent on the local temperatures, so evaluation of the temperature field during the mould filling is necessary in the simulation. Once the feedstock is considered as isotropic and the Fourier model for heat flux is applied, the heat transfer is controlled by the following advective-diffusive equation:

$$
\begin{aligned}
& \forall \mathbf{X} \in \Omega^{F}, \quad \rho_{p} C_{p}\left(\frac{\partial T}{\partial t}+\mathbf{V} \bullet \nabla T\right)=k_{p} \Delta T+\sigma_{p}^{\prime}: \dot{\epsilon}_{p} \\
& \forall \mathbf{X} \in \Omega^{V}, \quad \rho_{a} C_{a}\left(\frac{\partial T}{\partial t}+\mathbf{V} \bullet \nabla T\right)=k_{a} \Delta T+\sigma_{a}^{\prime}: \dot{\epsilon}_{a}
\end{aligned}
$$

where $T$ stands for the temperature field in the mould, $C_{p}$ and $k_{p}$ are the specific heat and thermal conductivity coefficient of feedstock, respectively. $C_{a}$ and $k_{a}$ are respectively the specific heat and thermal conductivity coefficient of air, $\sigma_{p}^{\prime}: \dot{\epsilon}_{p}$ and $\sigma_{a}^{\prime}: \dot{\epsilon}_{a}$ stand for the dissipations associated to the viscous flow of feedstock and the viscous air flow, $\dot{\epsilon}_{p}$ and $\dot{\epsilon}_{a}$ are the strain rate in filled and void portions.

\section{Solution procedure with a fully vectorial explicit strategy}

Instead of the use of MINI elements or reduced integration [11], the proposed algorithm is based on the elements with equal order interpolations. A smoothing procedure is systematically performed without piecewise operations to avoid mesh locking in incompressible flows. The interpolations of different field variables are written simply as:

$$
\{\mathbf{V}, P, F, T\}=\sum_{i=1}^{m} N_{i}\left\{\mathbf{V}_{i}, P_{i}, F_{i}, T_{i}\right\}
$$

in which $N_{i}$ stands for the interpolation functions, $m$ is the node number in an element, $T$ stands for the temperature.

In the explicit algorithm, the momentum conservation equations (1a) and (1b) can be split into two fractional steps: 1) solution for the viscous diffusion effect and 2) incompressibility condition maintaining.

\subsection{Viscous diffusion}

The fractional step for viscous diffusion is expressed as:

$$
\begin{aligned}
& \forall \mathbf{X} \in \Omega^{F}, \quad \rho_{f} \frac{\partial \mathbf{V}^{*}}{\partial t}=\nabla \bullet\left(\mu_{f} \dot{\epsilon}_{f}\right)+\mathbf{f}^{e x t} \\
& \forall \mathbf{X} \in \Omega^{V}, \quad \rho_{a} \frac{\partial \mathbf{V}^{*}}{\partial t}=\nabla \bullet\left(\mu_{a} \dot{\epsilon}_{a}\right)+\mathbf{f}^{e x t}
\end{aligned}
$$

where $\mathbf{V}^{*}$ stands for the intermediate values for solution of the velocity fields, $\mathbf{f}^{e x t}$ stands for the external loads.

Equations (6a) and (6b) are discretized by the standard GFEM procedure. It results in:

$$
\mathbf{M} \frac{\left(\mathbf{V}^{*}-\mathbf{V}_{n}\right)}{\Delta t}=\mathbf{F}^{\sigma}\left(\mathbf{V}_{n}\right)+\mathbf{F}^{e x t}
$$

In the above equations, $\mathbf{F}^{\sigma}$ represents the effects of viscous diffusion, $\mathbf{F}^{e x t}$ is the external load vector associated to gravity vector and the boundary conditions, $\mathbf{M}$ is a lumped mass matrix built as:

$$
\mathbf{M}=\underset{\Omega}{A} \int_{\Omega^{e}} \mathbf{N}^{T} \rho \mathbf{N} d \Omega^{e}
$$

in which $\mathbf{N}$ is the matrix of interpolation function at the element level. The mass matrix is lumped into diagonal form to get the solution uncoupled and explicit.

\subsection{Incompressibility}

To respect the incompressibility condition, the pressure field has to be solved in a global way in the previous algorithm. It makes the simulation very expensive in the case of large scale problems. To account such a drawback, a new method is proposed for maintaining the incompressibility condition. The intermediate velocity field, obtained by the fractional step for the viscous diffusion effect, is then corrected by a special factor $\lambda$. This factor is determined by the respect of incompressibility condition. It is a local operation in which each localized correction at element level is proportional to the deviation value with 
respect to incompressibility in the intermediate velocity field. However, a smoothing operation is necessary for the deviation values to avoid numerical instabilities, as only the elements with equal order interpolations are used.

In order to satisfy the incompressibility condition, the following equations must be dealt to correct the intermediate velocity fields:

$$
\begin{aligned}
& \forall \mathbf{X} \in \Omega^{F}, \rho_{f} \frac{\partial\left(\mathbf{V}_{n+1}-\mathbf{V}^{*}\right)}{\partial t}=-\nabla \lambda^{f}\left[\left(\nabla \cdot \mathbf{V}^{*}\right) \mathbf{I}\right], \\
& \forall \mathbf{X} \in \Omega^{V}, \rho_{a} \frac{\partial\left(\mathbf{V}_{n+1}-\mathbf{V}^{*}\right)}{\partial t}=-\nabla \lambda^{a}\left[\left(\nabla \cdot \mathbf{V}^{*}\right) \mathbf{I}\right] .
\end{aligned}
$$

In above equations, incompressibility condition in the filled part is accounted by the right hand term with a scaling factor $\lambda^{f}$.

These equations (9a) and (9b) are discretized by GFEM. The incompressible velocity field $\mathbf{V}_{n+1}$ is obtained directly from:

$$
\mathbf{M} \frac{\left(\mathbf{V}_{n+1}-\mathbf{V}^{*}\right)}{\Delta t}=\mathbf{F}^{p}\left(\mathbf{V}^{*}\right)
$$

where $\mathbf{V}_{n+1}$ and $\mathbf{V}^{*}$ represent the final incompressible and intermediate velocity fields, $\mathbf{F}^{p}$ stands for the correction term that is used to satisfy the incompressibility condition. This term is built as:

$$
\mathbf{F}^{\mathbf{p}}(\mathbf{V} *)=-\underset{\boldsymbol{\Omega}}{\mathbf{A}} \int_{\boldsymbol{\Omega}^{\mathbf{e}}} \mathbf{B}^{\mathbf{T}} \mathbf{I}^{\mathbf{e}} \lambda_{n+1} \bar{d}_{s m}\left(\mathbf{V}^{*}\right) d \Omega^{e}
$$

where $\mathbf{A}$ stands for the FEM assembly operator, $\bar{d}_{s m}\left(\mathbf{V}^{*}\right)$ is the values deviated from incompressibility for the intermediate velocity field $\mathbf{V}^{*}$ at each Gauss point, which is smoothened locally in the patch with its neighbour elements, $\mathbf{B}$ is the matrix for derivative of the interpolation functions used to calculate the strain rate in each element, $\mathbf{I}^{e}$ is a constant matrix, $\lambda_{n+1}$ is the regularization factor for filled and void domains respectively in the mould.

The dilatation value $d$ for the intermediate field $\mathbf{V}^{*}$ at each Gauss point is defined as:

$$
d\left(\mathbf{V}^{*}\right)=\frac{1}{3} \operatorname{tr}\left(\dot{\varepsilon}^{*}\right) .
$$

In the mixed formulations based on MINI elements for incompressible flows, the interpolation of velocity field is one order higher than that of the pressure field. Instead of it, the reduced integration can be used. As an alternative method in the new proposed algorithm, a smoothing operation is chosen to avoid the numerical instabilities. It is a systematic operation without piecemeal treatments [12]. The nodal values of the deviation term $d_{s m}$ are obtained from the relationship:

$$
\mathbf{M}_{\mathbf{0}} d_{s m}=\underset{\boldsymbol{\Omega}}{\mathbf{A}} \int_{\boldsymbol{\Omega}^{\mathbf{e}}} \mathbf{N}^{\mathbf{T}} d\left(\mathbf{V}^{*}\right) d \Omega^{e}
$$

in which $\mathbf{M}_{0}$ is a pseudo lumped mass matrix in diagonal form, built as:

$$
\mathbf{M}_{\mathbf{0}}=\underset{\boldsymbol{\Omega}^{\mathrm{e}}}{\mathbf{A}} \int_{\mathbf{N}^{\mathbf{T}}} \mathbf{N} \mathrm{d} \boldsymbol{\Omega}^{e}
$$
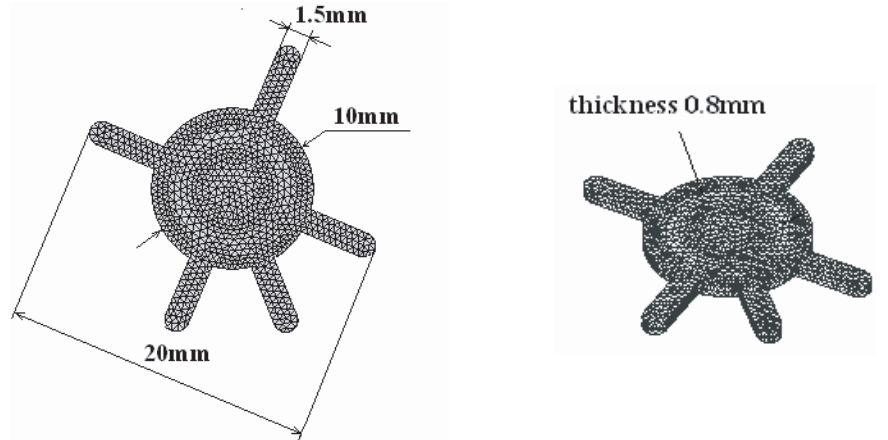

Fig. 4. 3D Mesh with tetrahedron elements (2521 nodes and 8620 elements).

So equation (11) can be written as:

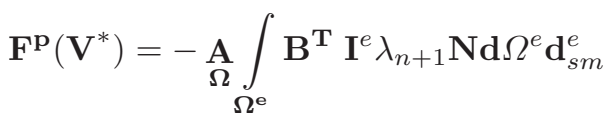

where the relationship $\bar{d}_{s m}=\mathbf{N} d_{s m}^{e}$ is applied at each Gauss points.

The same operations are necessary in the void mould part to maintain uniqueness of the solution model. In order to keep the numerical stability, the fictive factors are used for void mould part in the simulations.

\subsection{Determination of the parameter $\lambda$}

The determination of parameter $\lambda_{n+1}$ is a key procedure in new method. The average deviation value in the filled part $\Omega^{F}$ is used to determine the $\lambda_{n+1}$ value for filled part in each time step. The way to determine $\lambda_{n+1}$ is expressed as:

$$
\lambda_{n+1}=\frac{1}{\Delta t} \frac{\left\langle\mathbf{D V}^{*}\right\rangle_{\text {sum }}}{\left\langle\mathbf{D M}^{-1} \underset{\mathbf{\Omega}^{\mathbf{F}}}{\mathbf{A}} \int_{\mathbf{\Omega}^{\mathbf{e}}} \mathbf{B}^{T} \mathbf{I}^{e}\left(\nabla \bullet \mathbf{V}^{*}\right) d \Omega^{e}\right\rangle_{\text {sum }}}
$$

where the notation \langle\rangle$_{\text {sum }}$ denotes the sum of all nodal values in the filled part, $\mathbf{D}$ is a divergence operator. When $\lambda_{n+1}$ is obtained, it can be introduced in equations (10) and (11) to verify the incompressibility condition.

\subsection{Determination of filling state}

Equation (3) is discretized by the standard TaylorGalerkin procedure, which results in:

$$
\mathbf{M}_{\mathbf{0}} \frac{\left(\mathbf{F}_{n+1}-\mathbf{F}_{n}\right)}{\Delta t}=\left[\mathbf{K}^{a d}+\mathbf{K}^{d f}\right] \mathbf{F}_{n}
$$

where $\mathbf{F}_{n}$ and $\mathbf{F}_{n+1}$ are the filling state vectors at $t_{n}$ and $t_{n+1}$ instant respectively, $\mathbf{K}^{a d}$ stands for advection effect, $\mathbf{K}^{d f}$ diffusion effect. 
a
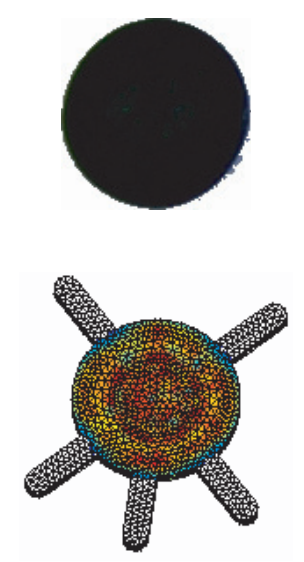

$63 \mathrm{~mm}^{3}$
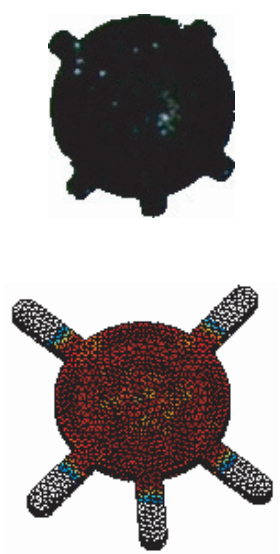

$68 \mathrm{~mm}^{3}$
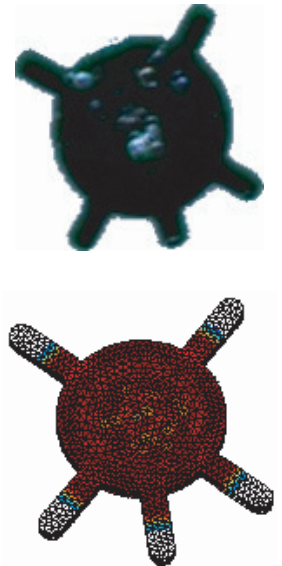

$73 \mathrm{~mm}^{3}$
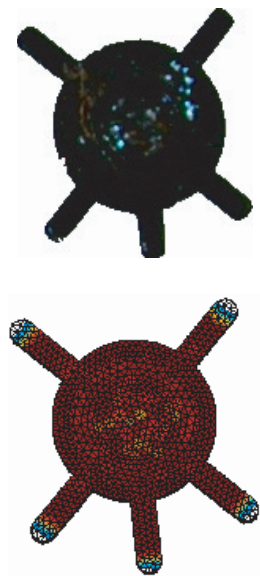

$78 \mathrm{~mm}^{3}$

(a) Experimental results

(b) simulation results

Fig. 5. Comparison of the front positions during mould filing (polypropylene temperature $220^{\circ} \mathrm{C}$, mould temperature $40{ }^{\circ} \mathrm{C}$, injection flux $\left.20 \mathrm{~mm}^{3} \cdot \mathrm{s}^{-1}\right)$.

\subsection{The calculation of heat transfer and temperature field}

The heat transfer in equations (4a) and (4a) are dealt with both advection and diffusion effects. These two effects are solved usually by an implicit solution but subjected to the limit on time steps [13]. A fractional step method proposed by Lewis [14] permits to evaluate the temperature field explicitly. The fractional advection and diffusion steps are written as:

$$
\begin{gathered}
\forall \mathbf{X} \in \Omega, \frac{\partial\left(T_{n+}-T_{n}\right)}{\partial t}=-\mathbf{V} \bullet \nabla T \\
\forall \mathbf{X} \in \Omega^{F}, \frac{\left(T_{n+1}-T_{n+}\right)}{\partial t}=\frac{1}{\rho_{p} C_{p}}\left(k_{p} \Delta T_{n+}+\sigma_{p}^{\prime}: \dot{\epsilon}_{p}\right) \\
\forall \mathbf{X} \in \Omega^{V}, \frac{\left(T_{n+1}-T_{n+}\right)}{\partial t}=\frac{1}{\rho_{a} C_{a}}\left(k_{a} \Delta T_{n+}+\sigma_{a}^{\prime}: \dot{\epsilon}_{a}\right)
\end{gathered}
$$

where $T_{n}$ and $T_{n+1}$ are the temperature fields at instant $t_{n}$ and $t_{n+1}, T_{n+}$ is an intermediate temperature field used in the fractional steps method.

Same as for the evaluation of filling state by TaylorGalerkin method, equation (18) can be discretized in the following form:

$$
\forall \mathbf{X} \in \Omega, \mathbf{M}_{0} \frac{\left(\mathbf{T}_{n+}-\mathbf{T}_{n}\right)}{\Delta t}=-\left[\mathbf{K}^{a d}(\mathbf{V})+\mathbf{K}^{d f}(\mathbf{V})\right] \mathbf{T}_{n}
$$

By Galerkin method, the solution of equations (19a) and (19b) can be written in the following manner:

$$
\forall \mathbf{X} \in \Omega, \mathbf{M}_{0} \frac{\left(\mathbf{T}_{n+1}-\mathbf{T}_{n+}\right)}{\Delta t}=\mathbf{K}^{t h} \mathbf{T}_{n+}+\dot{\mathbf{Q}}+\mathbf{q}^{c}
$$

where $\mathbf{K}^{t h}$ is the thermal diffusion term, $\mathbf{Q}$ is the heat dissipation term, $\mathbf{q}^{c}$ is the heat convection term.
Table 1. (a) Experimental results (b) simulation results.

\begin{tabular}{cc}
\hline Feedstock & polypropylene (PP, EP548N) \\
\hline Feedstock temperature & $220^{\circ} \mathrm{C}$ \\
Mould temperature & $40{ }^{\circ} \mathrm{C}$ \\
Injection flux & $20 \mathrm{~mm}^{3} \cdot \mathrm{s}^{-1}$ \\
Injection time & $1 \mathrm{~s}$ \\
Packing time & $10 \mathrm{~s}$ \\
\hline
\end{tabular}

\section{Numerical examples and experiments validations}

For validation of the numerical simulation results, the mould with five small channels in its cavity shown in Figure 2 is injected by polypropylene with two -colours Battenfeld microsystem 50. In the case, only one injection unit is used. In order to catch the filling front at different time steps, the mould cavity is intentionally injected with prescribed volumes of polymer to observe the filling process, as shown in Figure 5.

The cavity is meshed with 3D tetrahedron elements for the calculation of filling process by the vectorial explicit algorithm shown in Figure 4. The cavity consists of 2521 nodes and 8620 tetrahedron elements. The viscous behaviours of polypropylene shown in Figure 3 and the injection process parameters shown in table 1 are applied in the simulation. Sticky and slippery frictionless wall boundary conditions are imposed in the numerical simulations. For the case of sticky wall conditions, the five channels are difficult to be filled. The filling difficulty is related to the sticky wall conditions that may induce high shear rate in the channels. For the case of slippery wall conditions, the simulation results are globally verified by 
the experimental ones, shown in Figure 5. When $63 \mathrm{~mm}^{3}$ volume of the polymer is filled into the cavity, the circular part in the centre of the model is almost fully filled. When $68 \mathrm{~mm}^{3}$ volume filled, the five channels begin to be filled simultaneously. With the filling evolution in the channels, the filling fronts go ahead in the four channels when $73 \mathrm{~mm}^{3}$ and $78 \mathrm{~mm}^{3}$ polymer volume is filled into the cavity. The simulation results are rather consistent with the experiment results.

\section{Conclusion}

A fully explicit vectorial algorithm has been developed for efficient simulation of micro injection molding. A specially designed model is implemented for the analysis of wall boundary condition effects in the simulation of micro injection process. The experimental mould has been manufactured to perform micro injection experiments. The viscous behaviors of polypropylene have been tested for the application in the numerical simulation. By the comparisons between simulation results and experimental ones, it can be observed that the simulation with slippery wall conditions fits better the experiment results than that with sticky ones. This research work establishes a good base to further the research in micro injection and microMIM.

Acknowledgements. The research is funded by National Natural Science Fondation of China (10772154) and K.C. Wong Education Foundation, here I would like to say thanks for their supports.

\section{References}

1. T. Barriere, J.C. Gelin, G. Michel, M. Sahli, C. Quinard, Injection moulding of micro-parts: applications to microgears, 10th ESAFORM Conference on Material Forming. Zaragoza, Spain (April, 2007) p. 671-676

2. B. Sha, S. Dimov, C. Griffiths, M.S. Packianather, Investigation of micro injection moulding: factors affecting the replication quality. J. Mater. Process. Tech. 183, 284-296 (2007)
3. CG. Kukla, Micro injection Molding. Int. J. Forming Processes 4, 253-269 (2001)

4. T. Osada, K. Nishiyabu, Y. Karasaki, S. Tanaka, H. Miura, Investigations on the variation of feedstock properties in Micro MIM Products. In: PIM 2003, Powder Injection Molding. Pennsylvannia, USA (2003) p. 1-11

5. A.C. Rota, F. Petzoldt, P. Imgrund, Micromolding of advanced material combinations. In: PIM 2003, Powder Injection Molding. Pennsylvannia, USA (2003) p. 1-18

6. J.C. Gelin, T. Barriere, M.Dutilly, Experiments and computational modelling of metal injection molding for forming small parts. Annals of the CIRP 48, 179-182 (1999)

7. J.C. Gelin, T. Barriere, B. Liu, Mould design methods by experiment and numerical simulation in metal injection molding. J. Engineering Manufacture, Part B 126, 1533$1547(2002)$

8. Z. Cheng, T. Barriere, B. Liu, J.C. Gelin, An efficient vectorized approach for the simulation of $3 D$ processes in metal injection molding. J. of Steel and Grid materials supplement, 687-692 (2004)

9. Y.K. Shen, Y.J. Shie, W.Y. Wu, Extension method and numerical simulation of micro injection molding. International Communications in Heat and Mass Transfer 31. 795-804 (2004)

10. Z. Cheng, T. Barriere, B. Liu, J.C. Gelin, A new explicit simulation for mould filling with high viscous feedstock. J. of Solid Mechanics 17, 347-352 (2004)

11. T. Hughes, The finite element method, Linear static and dynamic finite element analysis. Prenctise-Hall: Englewood Cliffs (1987)

12. R.L. Lee, P.M. Gresho, R.L. Sani, Smoothing techniques for certain primitive variable solutions of the NavierStokes equations. Int. J. Numer. Meth. Eng. 14, 1785-1804 (1979)

13. A.S. Usmani, J.T. Cross, R.W. Lewis, A finite element model for the simulations of mould filling in metal casting and the associated heat transfer. Int. J. Numer. Meth. Eng. 35, 787-806 (1992)

14. R.W. Lewis, A.S. Usmani, T.J. Cross, Efficient mould filling simulation in castings by an explicit finite element method. Int. J. Numer. Meth. Fl. 20, 493-506 (1995) 